\title{
IMAGE SEGMENTATION MODEL USING ACTIVE CONTOUR AND IMAGE DECOMPOSITION
}

\author{
Xavier Bresson and Jean-Philippe Thiran \\ Signal Processing Institute (ITS), \\ Swiss Federal Institute of Technology of Lausanne (EPFL) \\ CH-1015 Lausanne, Switzerland \\ \{Xavier.Bresson, JP.Thiran\}@epfl.ch
}

\begin{abstract}
This paper proposes an image segmentation model based on the active contour model, the Mumford-Shah functional and the image decomposition process. Generally speaking, the active contour model detects boundaries in images from sharp intensities variations and the Mumford-Shah model finds smooth regions from homogeneous intensities. Our model merges these two complementary approaches while considering the Four Color Theorem to globally partition any given image. We also consider the textural part lying in natural images by separating it from the geometric part, which contains the meaningful objects, to help the segmentation process. Our segmentation model is experimented with a 1-D signal and 2-D images.
\end{abstract}

\section{INTRODUCTION}

One of the most fundamental issues in the fields of image processing and computer vision is image segmentation. It is the basis of higher level applications such as in medical imaging. Its objective is to determine a partition of an image into a finite number of semantically important regions. This paper proposes a new image segmentation model based on the active contour/snake model and the image decomposition process. More precisely, the main contributions of this paper are as follows:

1. definition of an image segmentation method based a global minimization of the active contour model,

2. integration of the image (structures-textures) decomposition process in the segmentation process,

3. definition of a method to determine an initial condition close to the optimal solution.

\section{RELATED WORKS}

\subsection{Image Segmentation Based on Active Contours}

Image segmentation consists of identifying homogeneous semantic regions in images. One way to carry out the segmentation process is to detect the boundaries between different semantic regions. This is realized with the active contour or snake model, initially proposed by Kass-Witkin-Terzopoulos in [1] and developed by Caselles-Kimmel-Sapiro in [2] and Kichenassamy-Kumar-OlverTannenbaum-Yezzi in [3]. The geodesic/geometric active contour (GAC) model is a variational model which consists of finding the curve $C$ which minimizes the following energy:

$$
E_{G A C}(C)=\int_{0}^{L(C)} g_{b}(|\nabla f(C(s))|) d s,
$$

where $d s$ is the Euclidean element of length, $L(C)$ is the length of the curve $C$ and the function $g_{b}$ is an edge indicator function that vanishes at object boundaries such as $g_{b}(|\nabla f|)=\frac{1}{1+\beta|\nabla f|^{2}}$, where $f$ is the original image and $\beta$ is an arbitrary positive constant. Hence, the energy functional (1) is actually a new length obtained by weighting the Euclidean element of length $d s$ by the function $g_{b}$ which contains information concerning the boundaries of objects [2]. The calculus of variations provides us the EulerLagrange equation of the functional $E_{G A C}$ and the gradient descent method gives us the flow that minimizes as fast as possible $E_{G A C}$ (see [2]). The evolution equation of active contour is handled with the level set method defined by Osher-Sethian [4], which efficiently solves the contour propagation problem and deal with topological changes.

Despite the many good numerical results obtained with this segmentation model and strong theoretical properties, the snake/ GAC model is highly sensitive to the initial condition. Actually, the quality of the segmentation result depends a lot on the choice of the initial contour, which means that a bad initial contour can give an unsatisfactory result. To overcome this drawback, several authors introduced region-based evolution criteria into active contour energy functionals built from intensity statistics and homogeneity requirements. One of the most successful models is the active contours without edges (ACWE) model developed by Chan-Vese [5]. The ACWE model is based on the Mumford-Shah (MS) model [6] which provides an optimal piecewise smooth approximation of a given image, in other words an image made up of homogeneous intensities regions which common boundaries are sharp and piecewise regular. The MS functional is defined as follows:

$$
\begin{aligned}
F_{M S}(s, C)= & \int_{\Omega}|s-f|^{2} d x+ \\
& \mu \int_{\Omega \backslash C}|\nabla s|^{2} d x+\nu \mathcal{H}^{N-1}(C),
\end{aligned}
$$

where $f$ is defined on a domain $\Omega, s$ corresponds to a piecewise smooth approximation of the original image $f, C$ is a discontinuity set (representing the edges of $s$ ), the length of $C$ is given by the $(N-1)$-dimensional Hausdorff measure $\mathcal{H}^{N-1}(C)$ (one can say that $\mathcal{H}^{1}$ is the length and $\mathcal{H}^{2}$ the area) and $\mu, \nu$ are positive parameters. The first term of (2) is a fidelity term w.r.t. the given data $f$, the second term is a regularization term that constraints the function $I$ to be smoothed inside the region $\Omega \backslash C$ and the last term imposes a regularization constraint on the discontinuity set $C$, i.e. the boundaries between smooth regions. The ACWE model proposes to minimize the Mumford-Shah functional, which is difficult to carry out in the original formulation, in the context of 
active contour, which is easier to realize with the calculus of variations. The ACWE model also corresponds to the piecewise constant/cartoon case of the MS functional obtained when $\mu \rightarrow \infty$. This case corresponds to the minimal partition problem, since the optimal solution is an image composed of regions of approximatively constant intensities equal to the mean value of intensities in the corresponding region. Finally, Vese-Chan considered in [7] the segmentation with the original Mumford-Shah energy (2).

As we said above, an usual problem when dealing the image segmentation with the active contour model is the local minima, which makes the initial guess critical to get satisfactory result. In a recent work, Chan-Esedoḡlu-Nikolova [8] propose an approach to overcome the limitation of local minima by determining a global minimum to the ACWE model. Inspired by this work, BressonEsedoğlu-Vandergheynst-Thiran-Osher proposed in $[9,10]$ a model to compute a global minimum to the standard snake model. In this paper, we propose to extend the result of $[9,10]$ to the general case of image segmentation based on the Four Color Theorem.

\subsection{Image Decomposition}

Image decomposition aims at splitting the structural/geometric part and the textural part lying in images. Structural parts are represented by piecewise smooth regions which constitutes the meaningful geometric components of images. Textural parts are, roughly speaking, fine scale-details, usually with some periodicity and oscillatory nature [11]. Meyer suggests in [12] to decompose an image $f$ into a component $s$ belonging to the space of functions with bounded variation, $B V$, and a component $t$ in the Banach space $G$ containing signals with large oscillations s.a. textures and noise. The variational model of Meyer is as follows:

$\min _{(s, t) \in B V \times G / f=s+t}\left\{F_{M}(s, t, \lambda)=\int_{\Omega}|\nabla s| d x+\lambda\|t\|_{G}\right\}$,

Since Meyer, several variational approaches, based on partial differential equations (PDEs), have been proposed to carry out the image decomposition task. In this paper, we propose to introduce the image decomposition process in the segmentation process to improve its performance. Indeed, the separation of the textural part and the geometric part will help us to segment the meaningful regions which boundaries are easily visible in the geometric part. The snake model will also help us to split the smooth part and the textural part, which can be used for other high-level processing tasks.

\section{GENERAL IMAGE SEGMENTATION MODEL}

We propose to compute a global minimum for the image segmentation model defined by Vese-Chan in [7]. More precisely, a global minimum for the active contour model based on the general formulation of the image segmentation method of MS is determined. The MS model is very well adapted to segment smooth regions lying in images but it does not take into account textures in its original definition. One of the motivation of our method is to consider textures in the MS model. In [7], Vese-Chan minimize the MS energy (2) using a multiphase level set approach motivated by the Four Color Theorem [13]. They use two level set functions to represent four phases (and triple junctions) and these four phases are sufficient to partition an image in a general way because each phase can be used to "color"/delimit different adjacent regions in an image according to the Four Color Theorem (see Figure 1).

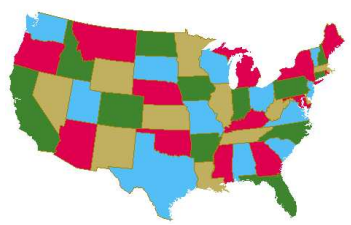

Fig. 1. Illustration of the Four Color Theorem.

The variational model introduced in [7] to approximate the general MS functional is:

$$
\begin{gathered}
\min _{\Omega_{C_{1}}, \Omega_{C_{2}}, s_{i j}}\left\{E_{V C}\left(\Omega_{C_{1}}, \Omega_{C_{2}}, s_{i j}, \lambda, \eta\right)=\sum_{m=1,2} \operatorname{Per}\left(\Omega_{C_{m}}\right)\right. \\
\left.+\lambda \sum_{i, j=+,-} \int_{\Omega_{i j}}\left(\eta\left(s_{i j}(x)-f(x)\right)^{2}+\left|\nabla s_{i j}(x)\right|^{2}\right) d x\right\},
\end{gathered}
$$

where $\Omega_{C_{m}}$ are two closed subsets of the image domain $\Omega, \lambda, \eta$ are two non-negative parameters, Per is the perimeter, $f$ is the given image and functions $s_{i j}$ represent four phases, defined in $\Omega_{i j} \subset \Omega$, to partition any given image s.t. $\Omega=\cup_{i j} \Omega_{i j}$ and $\cap_{i j} \Omega_{i j}=\emptyset$.

At this stage, we replace the $L^{2}$-norm of the fidelity term in the energy (4) by the $L^{1}$-norm to separate textures from the structural parts. The gradient-based term in (4) is unchanged to capture smooth regions. Thus, the energy (4) becomes:

$$
\begin{gathered}
E^{1}\left(\Omega_{C_{1}}, \Omega_{C_{2}}, s_{i j}, \lambda, \eta\right)=\sum_{m=1,2} \operatorname{Per}\left(\Omega_{C_{m}}\right)+ \\
\lambda \sum_{i, j=+,-} \int_{\Omega_{i j}}\left(\eta\left|s_{i j}-f\right|+\left|\nabla s_{i j}\right|^{2}\right) d x
\end{gathered}
$$

Minimizing (5) w.r.t. the functions $s_{i j}$ leads to the variational problem for each function $s_{i j}$ :

$$
\min _{s_{i j}}\left\{\int_{\Omega_{i j}} \eta\left|s_{i j}-f\right|+\left|\nabla s_{i j}\right|^{2} d x\right\} .
$$

A new function $t_{i j}$ is introduced in the previous minimization problem to extract textures:

$\min _{s_{i j}, t_{i j}}\left\{\int_{\Omega_{i j}} \eta\left|t_{i j}\right|+\frac{1}{2 \theta_{s}}\left(s_{i j}-\left(f-t_{i j}\right)\right)^{2}+\left|\nabla s_{i j}\right|^{2} d x\right\}$,

where the parameter $\theta_{s}>0$ is small so that we almost have $f=$ $s_{i j}+t_{i j}$ in $\Omega_{i j}$. Minimizing (7) w.r.t. the function $s_{i j}$, using the calculus of variations, leads to:

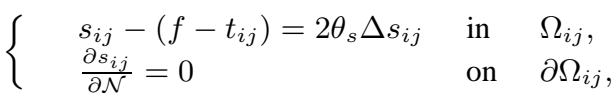

and minimizing (7) w.r.t. the function $t_{i j}$ gives:

$$
t_{i j}=\left\{\begin{array}{lll}
f-s_{i j}-\theta_{s} \eta & \text { if } & f-s_{i j} \geq \theta_{s} \eta \\
f-s_{i j}+\theta_{s} \eta & \text { if } & f-s_{i j} \leq \theta_{s} \eta \\
0 & \text { if } & \left|f-s_{i j}\right| \leq \theta_{s} \eta
\end{array}\right.
$$

In [7], regions $s_{i j}$ are represented by two level set function, namely $\phi_{1}, \phi_{2}$, s.t.

$$
s(x):=\left\{\begin{array}{lll}
s_{++}(x) & \text { if } & \phi_{1}(x)>0, \phi_{2}(x)>0, \\
s_{+-}(x) & \text { if } & \phi_{1}(x)>0, \phi_{2}(x)<0, \\
s_{-+}(x) & \text { if } & \phi_{1}(x)<0, \phi_{2}(x)<0, \\
s_{--}(x) & \text { if } & \phi_{1}(x)<0, \phi_{2}(x)>0,
\end{array}\right.
$$


Thus, Energy (5) can be re-written as follows:

$$
\begin{array}{r}
E^{2}\left(\phi_{1}, \phi_{2}, s_{i j}, t_{i j}, \lambda, \eta, \theta_{s}\right)=\sum_{m=1,2} \int_{\Omega}\left|\nabla H_{\epsilon}\left(\phi_{m}\right)\right|+ \\
\lambda \sum_{i, j=+,-} \int_{\Omega}\left(\eta\left|t_{i j}\right|+\frac{1}{2 \theta_{s}}\left(s_{i j}-\left(f-t_{i j}\right)\right)^{2}+\right. \\
\left.\left|\nabla s_{i j}\right|^{2}\right) H\left(i \phi_{1}\right) H\left(j \phi_{2}\right) d x,
\end{array}
$$

The flow minimizing the energy (11) is as follows:

$\partial_{t} \phi_{m}=H^{\prime}\left(\phi_{m}\right)\left\{\operatorname{div}\left(\frac{\nabla \phi_{m}}{\left|\nabla \phi_{m}\right|}\right)-\lambda r_{m}\left(x, s_{i j}, t_{i j}, \eta, \theta_{s}\right)\right\}$

where

$$
\begin{array}{r}
r_{m}\left(x, s_{i j}, t_{i j}, \eta, \theta_{s}\right)=\sum_{i, j=+,-}(-i)^{2-m}(-j)^{2-n}\left(\eta\left|t_{i j}\right|+\right. \\
\left.\frac{1}{2 \theta_{s}}\left(s_{i j}-\left(f-t_{i j}\right)\right)^{2}+\left|\nabla s_{i j}\right|^{2}\right) H\left(j \phi_{n}\right)
\end{array}
$$

for $m, n=1,2$ and $m \neq n$. If a non-compactly supported smooth approximation of the Heaviside function is chosen, the steady state solution of the gradient flow (11) is the same as:

$$
\partial_{t} \phi_{m}=\operatorname{div}\left(\frac{\nabla \phi_{m}}{\left|\nabla \phi_{m}\right|}\right)-\lambda r_{m}\left(x, s_{i j}, t_{i j}, \eta, \theta_{s}\right)
$$

and this equation is the gradient descent flow of the energy:

$$
\begin{aligned}
& E^{3}\left(\phi_{1}, \phi_{2}, s_{i j}, \lambda, \eta, \theta_{s}\right)=\sum_{m=1,2} \int_{\Omega}\left|\nabla \phi_{m}\right|+ \\
& \lambda \int_{\Omega} r_{m}\left(x, s_{i j}, t_{i j}, \eta, \theta_{s}\right) \phi_{m} d x .
\end{aligned}
$$

As a result of the previous developments, the following constrained minimization model is proposed to carry out the general image segmentation process:

$$
\begin{gathered}
\min _{0 \leq u_{m} \leq 1}\left\{E_{m}\left(u_{m}, s_{i j}, t_{i j}, \lambda, \eta, \theta_{s}\right)=\sum_{m=1,2} \int_{\Omega} g_{b}(x)\left|\nabla u_{m}\right|\right. \\
\left.+\lambda \int_{\Omega} r_{m}\left(x, s_{i j}, t_{i j}, \eta, \theta_{s}\right) u_{m} d x\right\}
\end{gathered}
$$

where $g_{b}$ is the edge detector function in the GAC model. The energy (16) provides us a global minimization for the active contour model. The global minimization theorem is the same as in $[9,10]$. Firstly, it consists of applying the coarea formula to notice that $\int_{\Omega} g_{b}(x)\left|\nabla\left(u_{m}=\mathbf{1}_{\Omega_{C_{m}}}\right)\right|=\int_{C_{m}} g_{b} d s=E_{G A C}\left(C_{m}\right)$ as in (1). Then, for fixed functions $u_{n \neq m}, s_{i j}, t_{i j}$, a theorem establishes that $\mathbf{1}_{\Omega_{C_{m}}(\mu)=\left\{x: u_{m}(x)>\mu\right\}}$ for $\mu \in[0,1]$ is a global minimizer of (16).

The snake variational model proposes in this framework is globally minimized, which is very important because a global minimization allows us to be independent of the initial condition. However, the proposed model is globally minimized only w.r.t. the snakes, represented by $u_{1}, u_{2}$, but not w.r.t. the functions $s_{i j}, t_{i j}$. Thus, the choice of the initial functions $s_{i j}, t_{i j}$ is critical to get a satisfactory segmentation result. The next section proposes a fast way to compute initial $s_{i j}, t_{i j}$ close to the optimal solution.

\section{INITIAL CONDITION}

We propose to determine a good initial condition for the functions $s_{i j}, t_{i j}$ to find the optimal segmentation solution. Two steps are followed:

1. Computation of an initial smooth function $s_{0}$ and an initial texture function $t_{0}$ as follows:

$$
\min _{s_{0}, t_{0}}\left\{\int_{\Omega} \eta\left|t_{0}\right|+\frac{1}{2 \theta_{s}}\left(s_{0}-\left(f-t_{0}\right)\right)^{2}+\left|\nabla s_{0}\right|^{2} d x\right\} .
$$

2. Determination of a function $s_{1}$ from $s_{0}$ with a region growing algorithm based on the Mumford-Shah model as described in [14].

The initialization process takes less than one minute for the 2-D images presented in the next section.

\section{RESULTS}

Firstly, our segmentation model is tested on the 1-D signal (Figure 2). Our model correctly detects the transitions between smooth regions (see small red circles). The decomposition between smooth (green curve) and textural parts (blue curve) provides us a good approximation of the original signal (black curve).

Secondly, our model is experimented on Figure 3 which is a linear combination of a smooth image, which intensities vary between $[0,1]$, and a texture image, varying between $[-0.6,0.6]$. The image also contains a triple junction which needs three phases to be detected. Our model is able to find the triple junction, the original smooth part and the texture part. The segmentation takes a few minutes to converge.

Finally, the image segmentation model is tested on the benchmark image Barbara which contains textures. The model recovers the smooth part of the given image and also the textures and several meaningful boundaries. As previously, a few minutes are needed to converge to the solution.

\section{REFERENCES}

[1] M. Kass, A. Witkin, and D. Terzopoulos, "Snakes: Active Contour Models," International Journal of Computer Vision, pp. 321-331, 1987.

[2] V. Caselles, R. Kimmel, and G. Sapiro, "Geodesic Active Contours," International Journal of Computer Vision, vol. 22(1), pp. 61-79, 1997.

[3] S. Kichenassamy, A. Kumar, P. Olver, A. Tannenbaum, and A.J. Yezzi, "Conformal Curvature Flows: From Phase Transitions to Active Vision," in Archive for Rational Mechanics and Analysis, 1996, vol. 134, pp. 275-301.

[4] S. Osher and J.A. Sethian, "Fronts Propagating with Curvature-Dependent Speed: Algorithms Based on Hamilton-Jacobi Formulations," Journal of Computational Physics, vol. 79(1), no. 12-49, 1988.

[5] T.F. Chan and L.A. Vese, "Active Contours Without Edges," IEEE Transactions on Image Processing, vol. 10(2), pp. 266277, 2001.

[6] D. Mumford and J. Shah, "Optimal Approximations of Piecewise Smooth Functions and Associated Variational Problems," Communications on Pure and Applied Mathematics, vol. 42, pp. 577-685, 1989. 

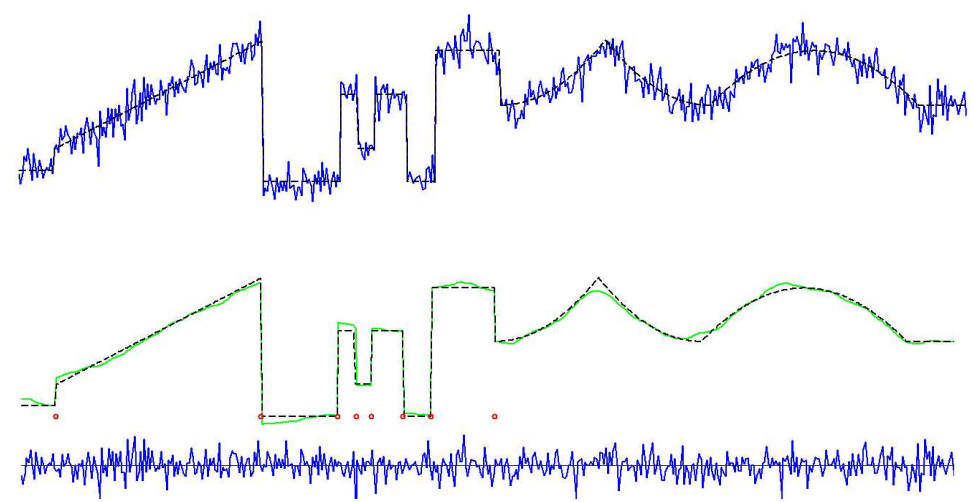

Fig. 2. Our segmentation model applied to a noisy 1-D signal. Figure (a): original signal in dark, noisy signal in blue. Figure (b): original signal in dark, active contour in red circles, smooth part in green, textural part in blue.

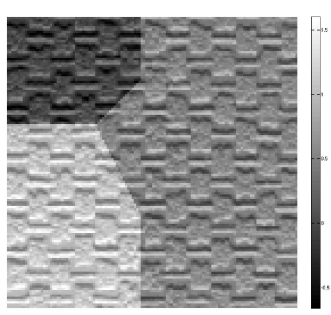

(a) Original Image.

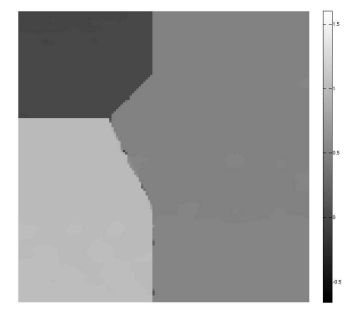

(c) Smooth/Structural Part of (a).

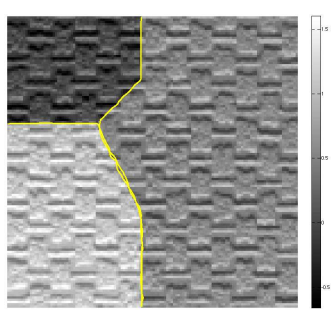

(b) Final Active Contour/ Boundaries of (a).

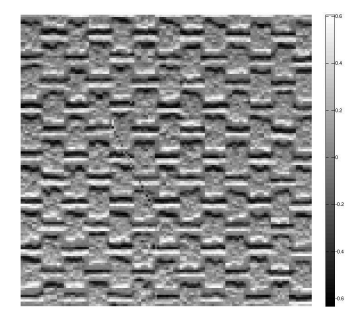

(d) Textural Part of (a).
Fig. 3. Segmentation of a triple junction.

[7] L.A. Vese and T.F. Chan, "A Multiphase Level Set Framework for Image Segmentation Using the Mumford and Shah Model," International Journal of Computer Vision, vol. 50(3), pp. 271-293, 2002.

[8] T.F. Chan, S. Esedoḡlu, and M. Nikolova, "Algorithms for Finding Global Minimizers of Image Segmentation and Denoising Models, UCLA CAM Report 04-54," 2004.

[9] X. Bresson, S. Esedoḡlu, P. Vandergheynst, J.-P. Thiran, and S. Osher, "Global Minimizers of The Active Contour/Snake Model, UCLA CAM Report 05-04,” 2005.

[10] X. Bresson, S. Esedoḡlu, P. Vandergheynst, J.-P. Thiran, and S. Osher, "Fast Global Minimization of the Active Contour/Snake Model," Journal of Mathematical Imaging and Vision (Submitted), 2005.

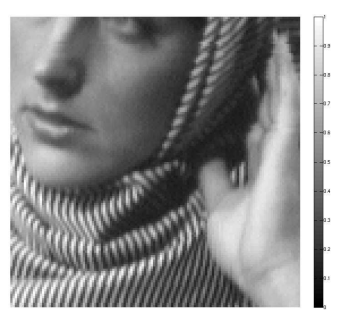

(a) Original Image.

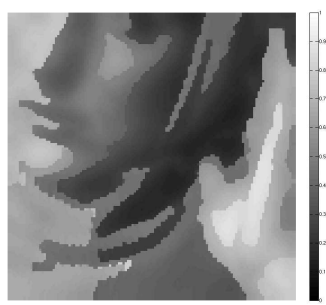

(c) Smooth/Structural Part of (a).

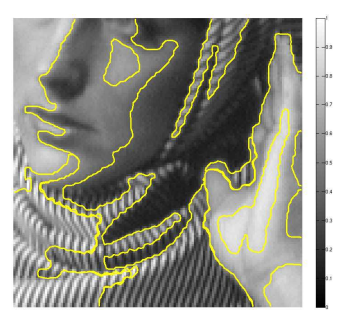

(b) Final Active Contour/ Boundaries of (a).

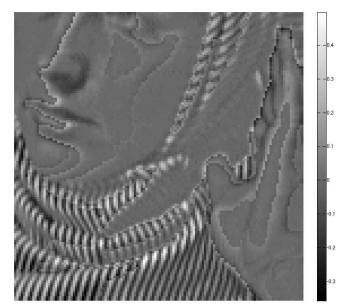

(d) Textural Part of (a).
Fig. 4. Segmentation of Barbara.

[11] J.-F. Aujol, G. Gilboa, T. Chan, and S. Osher, "StructureTexture Image Decomposition - Modeling, Algorithms, and Parameter Selection," International Journal of Computer Vision, vol. (in press), 2005.

[12] Y. Meyer, Oscillating Patterns in Image Processing and in Some Nonlinear Evolution Equations, The Fifteenth Dean Jacquelines B. Lewis Memorial Lectures, 2001.

[13] R. Fritsch and G. Fritsch, The Four-Color Theorem. History, Topological Foundations and Idea of Proof, Springer, 1998.

[14] A. Petrovic and P. Vandergheynst, "Multiscale Variational Approach to Simultaneous Image Regularization and Segmentation," in International Symposium on Image and Signal Processing and Analysis, 2003. 